\title{
Two-Photon Interactions with Nuclear Breakup in Relativistic Heavy Ion Collisions
}

Anthony J. Baltz, Yuri Gorbunov, Spencer R. Klein and Joakim Nystrand

\section{August 26, 2009}

This work was supported by the Director, Office of Science, Office of Nuclear Science of the U.S. Department of Energy under Contract No. DE-AC02$05 C H 11231$. 


\title{
Two-Photon Interactions with Nuclear Breakup in Relativistic Heavy Ion Collisions
}

\author{
Anthony J. Baltz ${ }^{1}$, Yuri Gorbunov ${ }^{2}$, Spencer R. Klein ${ }^{3}$ and Joakim Nystrand ${ }^{4}$ \\ ${ }^{1}$ Brookhaven National Laboratory, Upton, NY, 11973, USA \\ ${ }^{2}$ Physics Department, Creighton University, Omaha, NE, 68178 \\ ${ }^{3}$ Lawrence Berkeley National Laboratory, Berkeley, CA 94720, USA \\ ${ }^{4}$ Dept. of Physics and Technology, \\ University of Bergen, Bergen, Norway
}

\begin{abstract}
Highly charged relativistic heavy ions have high cross-sections for two-photon interactions. The photon flux is high enough that two-photon interactions may be accompanied by additional photonuclear interactions. Except for the shared impact parameter, these interactions are independent. Additional interactions like mutual Coulomb excitation are of experimental interest, since the neutrons from the nuclear dissociation provide a simple, relatively unbiased trigger.

We calculate the cross sections, rapidity, mass and transverse momentum $\left(p_{T}\right)$ distributions for exclusive $\gamma \gamma$ production of mesons and lepton pairs, and for $\gamma \gamma$ reactions accompanied by mutual Coulomb dissociation. The cross-sections for $\gamma \gamma$ interactions accompanied by multiple neutron emission $(X n X n)$ and single neutron emission $(1 n 1 n)$ are about 1/10 and 1/100 of that for the unaccompanied $\gamma \gamma$ interactions. We discuss the accuracy with which these cross-sections may be calculated. The typical $p_{T}$ of $\gamma \gamma$ final states is several times smaller than for comparable coherent photonuclear interactions, so $p_{T}$ may be an effective tool for separating the two classes of interactions.
\end{abstract}

PACS numbers: PACS Numbers: 25.20.-x, 12.40.Vv, 13.60.Le 


\section{INTRODUCTION}

With their large charges $Z$, relativistic heavy ions carry strong electromagnetic fields which act as strong sources of nearly-real photons. These photons can induce a wide variety

of photonuclear and two-photon physics [1]. The photon flux scales as $Z^{2}$, so the two-photon cross-section scales as $Z^{4}$. The particle production rates are competitive with those obtained at $e^{+} e^{-}$colliders. At the LHC, there is strong interest in using two-photon physics to search for signs of new physics [2].

Because the coupling constant $Z \alpha \approx 0.6(\alpha \approx 1 / 137$ is the electromagnetic coupling constant) is large, two-photon interactions may be accompanied by additional electromagnetic interactions, such as additional two-photon reactions, or photonuclear interactions. Photonuclear reactions can produce collective nuclear excitations, e.g. a Giant Dipole Resonance (GDR), or can involve more energetic processes, such as pion production. Multiple additional reactions also occur. For example, one photon from each nucleus can dissociate the other, in mutual Coulomb dissociation (MCD) [3, 4]. Experimentally, neutrons from the nuclear dissociation are detected in zero degree calorimeters (ZDCs) downstream from the collision points, in both directions. These neutrons make a convenient trigger, separating two-photon and other ultra-peripheral collisions (UPCs) from backgrounds such as cosmic rays and beam-gas interactions. The neutrons also 'tag' events with a smaller mean impact parameter than exclusive two-photon events. Neutron tagging has been used to study $e^{+} e^{-}$ [5, 6] and vector meson photoproduction [7, 8, 9].

Tagging is of special interest for $e^{+} e^{-}$because the stronger fields at small impact parameters, where non-perturbative or strong-field electrodynamic effects will be strongest. Calculations of $e^{+} e^{-}$pair production accompanied by MCD are challenging, because the typical impact parameters are smaller than the electron Compton wavelength, $\lambda_{C}=386 \mathrm{fm}$ [10]. The cross-section measured by STAR is larger than lowest-order QED calculation; the difference can be explained by higher order corrections [11].

Here, we calculate the cross sections, rapidity, invariant mass, and transverse momentum distributions for two-photon production of a selection of scalar and tensor mesons and $\mu^{+} \mu^{-}$ and $\tau^{+} \tau^{-}$pairs. We calculate the cross-sections and distributions with and without nuclear breakup, and explore how nuclear breakup affects the reactions.

Because the cross-section for n-photon interactions scales as $Z^{2 n}$ we focus on heavy nuclei: 


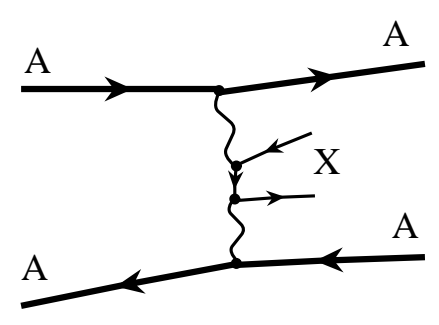

a)

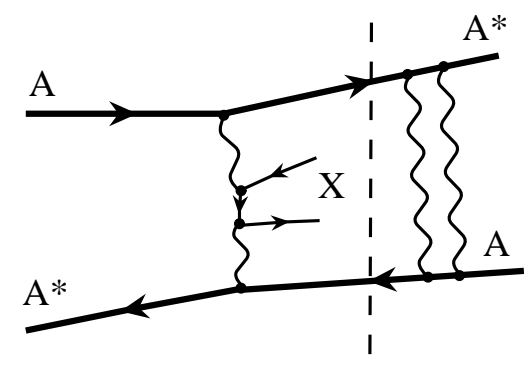

b)

FIG. 1: The dominant Feynman diagrams for two photon reactions (a) without and (b) with nuclear excitation. Cross diagrams and, for (b) time reversed diagrams are omitted; due to factorization, they simply add.

gold-gold collisions at a center of mass energy of $\sqrt{s_{n n}}=200 \mathrm{GeV}$ per nucleon (beam Lorentz boost $\gamma=108$ ), as seen at the Relativistic Heavy Ion Collider (RHIC) at Brookhaven National Laboratory, and lead-lead collisions at an energy of $5.5 \mathrm{TeV}$ per nucleon, as will be produced at the large hadron collider (LHC) at CERN, where $\gamma=2940$. We assume that RHIC runs for $10^{7} \mathrm{~s} /$ year, at an average gold-gold luminosity of $2 \times 10^{26} / \mathrm{cm}^{2} / \mathrm{s}$ [12]. The LHC is expected to devote $\sim 1$ month, or $10^{6} \mathrm{~s} /$ year to lead beams, at an average luminosity of $10^{27} / \mathrm{cm}^{2} / \mathrm{s}$ [12].

Section II will present a calculation of the two-photon luminosity. Section III discusses the effects of multiple interactions, while Section IV finds the two-photon luminosity under various different nuclear breakup conditions and discuss the uncertainties in the calculation. The final state $p_{T}$ spectra are discussed in Section V, and Section VI gives some conclusions.

\section{TWO-PHOTON LUMINOSITY}

According to the method of equivalent photons, the cross section for a two-photon reaction, Fig. 1a, factorizes into an elementary cross section for $\gamma \gamma \rightarrow X$ and a $\gamma \gamma$ luminosity $\mathcal{L}_{\gamma \gamma}[13,14]$. The cross section to produce a final state with mass $W$ is

$$
\sigma(A+A \rightarrow A+A+X)=\int d k_{1} d k_{2} \frac{n\left(k_{1}\right)}{k_{1}} \frac{n\left(k_{2}\right)}{k_{2}} \sigma(\gamma \gamma \rightarrow X(W))
$$

where $k_{1}$ and $k_{2}$ are the two photon energies, and $n(k)$ is the photon flux at energy $k$.

The $\gamma \gamma$ luminosity is given by convolution of the equivalent photon spectra from the 
two nuclei. In impact parameter space, the total number of photons from one nucleus is obtained by integrating over all impact parameters larger than some minimum, typically given by the nuclear radius. This is similar to integrating over all possible momentum transfers, $Q$, from some minimum determined by the kinematics up to a maximum given by the nuclear form factor. In hadronic collisions, the impact parameter representation provides the best way to incorporate effects of strong absorption. Hadronic interactions will dominate in collisions where both hadronic and electromagnetic interactions are possible. The hadronic interaction must therefore be excluded to obtain the effective or usable cross section for two-photon interactions. In impact parameter space, this can be accomplished by requiring that the impact parameter be larger than the sum of the nuclear radii. The equivalent two-photon luminosity is thus [15, 17]:

$$
\frac{d \mathcal{L}_{\gamma \gamma}}{d W d y}=\mathcal{L}_{A A} \frac{W}{2} \int_{b_{1}>R_{A}} d^{2} b_{1} \int_{b_{2}>R_{A}} d^{2} b_{2} n\left(k_{1}, b_{1}\right) n\left(k_{2}, b_{2}\right) \Theta\left(\left|\overrightarrow{b_{1}}-\overrightarrow{b_{2}}\right|-2 R_{A}\right)
$$

where $\mathcal{L}_{A A}$ is the ion-ion luminosity, $n(k, b)$ is the flux of photons with energy $k$ at impact parameter $b$, and $R_{A}$ is the Woods-Saxon nuclear radius.

The requirements $b_{1}>R_{A}$ and $b_{2}>R_{A}$ ensure that the final state is produced outside the nuclei. Otherwise, the final state will usually interact with the nucleus, destroying itself and breaking up the nucleus. This requirement may not be strictly necessary for some final states, such as lepton pairs. Alternately, a smaller radius might be appropriate. However, since the electric fields drop rapidly for $b<R_{A}$, this is a relatively small correction. The $\Theta$ function imposes a requirement that the nuclei not physically collide; we discuss more detailed hadronic interaction models below.

The photon flux may be modelled using the Weizsäcker-Williams method. For $\gamma \gg 1$

$$
n(k, b)=\frac{d^{3} N}{d k d^{2} b}=\frac{Z^{2} \alpha}{\pi^{2} k b^{2}} x^{2} K_{1}^{2}(x)
$$

where $x=b k / \gamma$. Here, $K_{1}$ is a modified Bessel function. The two photon energies $k_{1}$ and $k_{2}$ determine the center of mass energy $W$ and rapidity $y$ :

$$
k_{1,2}=\frac{W}{2} e^{ \pm y}
$$

and

$$
y=1 / 2 \ln \left(k_{1} / k_{2}\right)
$$


The maximum effective two-photon energy, $W_{\max }$ occurs at $y=0$, when $k_{1}=k_{2}=\gamma / R_{A}$. $W_{\max }$ is about $6 \mathrm{GeV}$ for gold at RHIC, and $150 \mathrm{GeV}$ for lead at the LHC. $W_{\max }$ is higher for lighter nuclei and protons, but the $\gamma \gamma$ luminosity per collision is lower, and multi-photon interactions are very rare.

Equation (2) treats the nuclei as hard spheres with radius $R_{A}$. Since there is a finite probability to have hadronic interactions at impact parameters $b>2 R_{A}$, more accurate hadronic interaction probabilities can be included by modifying Eq. (2), to

$$
\frac{\mathcal{L}_{\gamma \gamma}}{d W d y}=\mathcal{L}_{A A} \frac{W}{2} \int_{b_{1}>R_{A}} d^{2} b_{1} \int_{b_{2}>R_{A}} d^{2} b_{2} n\left(k_{1}, b_{1}\right) n\left(k_{2}, b_{2}\right)\left[1-P_{H}\left(\left|\vec{b}_{1}-\vec{b}_{2}\right|\right)\right]
$$

with the hadronic interaction probability

$$
P_{H}(\vec{b})=1-\exp \left(-\sigma_{n n} \int d^{2} \vec{r} T_{A}(\vec{r}) T_{A}(\vec{r}-\vec{b})\right) .
$$

$\sigma_{n n}$ is the total hadronic interaction cross section, $52 \mathrm{mb}$ at RHIC and $88 \mathrm{mb}$ at the LHC [12]. We use the total cross sections, since even an elastic nucleon-nucleon interaction will break up the nucleus. The nuclear thickness function is the integral of the nuclear density, $\rho(r)$

$$
T_{A}(\vec{b})=\int d z \rho(\vec{b}, z) d z
$$

where $\vec{b}$ is the impact parameter from the center of the nucleus. The nuclear density profile $\rho\left(r=\sqrt{|\vec{b}|^{2}+z^{2}}\right)$ of heavy nuclei is well described with a Woods-Saxon distribution. We use parameters determined from electron scattering data $(\mathrm{R}=6.38 \mathrm{fm}$ for $\mathrm{Au}$ and $\mathrm{R}=6.62$ fm for $\mathrm{Pb})[12]$.

As Fig. 2 shows, Eq. (6) gives $\gamma \gamma$ luminosities about $5 \%$ lower than the hard sphere model for $W=0.1 W_{\max }$, falling to $15 \%$ lower for $W=W_{\max }[15]$. These differences are comparable to those found elsewhere [16].

Baur and Ferrara-Filho derived Eq. (2), and then used a change of variables and the $\Theta$ function to reduce the dimensions of the integral [17]. Although this approach speeds the calculation, it works poorly for the realistic models of nuclear density or when additional photon exchange is included. Cahn and Jackson used a related approach, calculating the luminosity analytically without the requirement $b>2 R_{A}$, and then numerically calculating a correction for the overlap [18]. This approach also cannot accomodate nuclear breakup.

In principle, the meson or lepton pair production cross section depends on the angle between $\vec{b}_{1}$ and $\vec{b}_{2}$, with different cross sections for parallel and perpendicular photon po- 


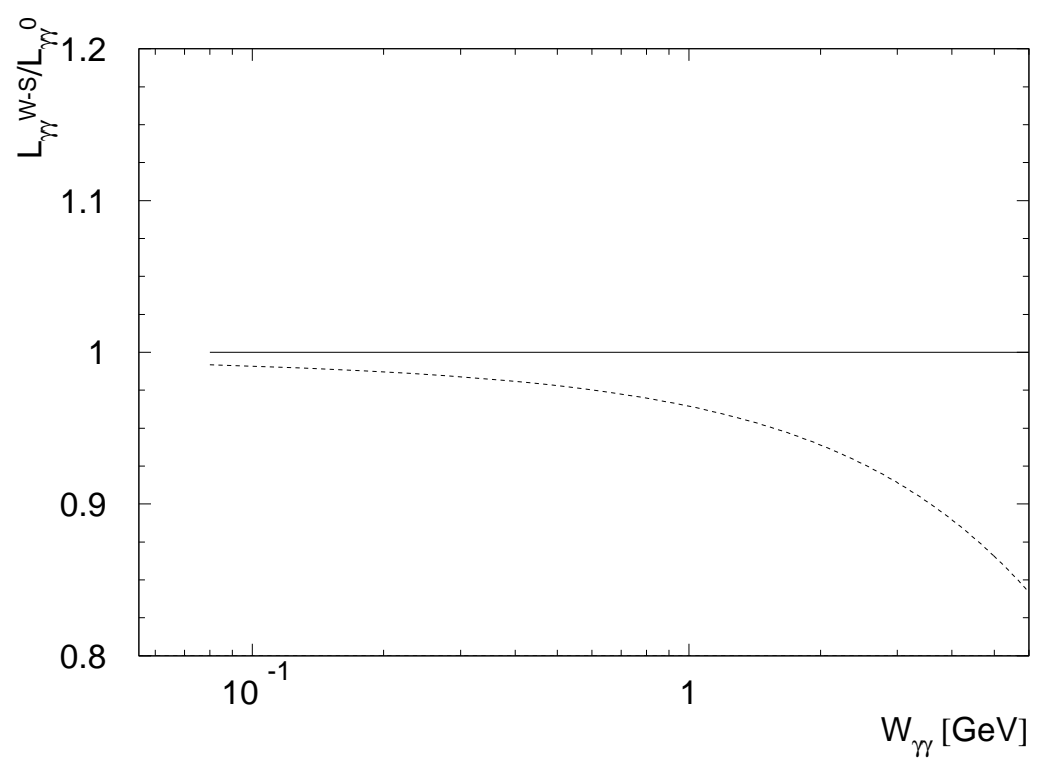

FIG. 2: Reduction in two-photon luminosity when the nucleon density is approximated with a Woods-Saxon distribution (dotted line) as compared with a flat distribution for hard sphere nuclei, requiring $\left|\overrightarrow{b_{1}}-\overrightarrow{b_{2}}\right|>2 R_{A}$ (solid line). The calculation is for gold-gold interactions at RHIC.

larizations [19]. However, after integration over $\vec{b}_{1}$ and $\vec{b}_{2}$, the differences are small, and we neglect them.

The cross section to produce a narrow resonance with spin $J$ and mass $m$ is

$$
\sigma_{\gamma \gamma}=8 \pi^{2}(2 J+1) \frac{\Gamma_{\gamma \gamma}}{2 W^{2}} \delta(W-m)
$$

where we neglect the width of the hadronic resonances. The magnitude of the error due to this narrow-resonance approximation scales linearly with the width. For coherent photonuclear $\rho^{0}$ production, including the width reduces the cross section by about $5 \%$ [12]. A similar correction is expected for two-photon production; these adjustments should should scale linearly with the relative width $(\Gamma / M)$ of the resonance.

We also consider the production of continuum lepton pairs. For leptons with mass $M$, the cross section is given by the Breit-Wheeler formula

$$
\sigma_{\gamma \gamma}=\frac{4 \pi \alpha^{2}}{W^{2}}\left[\left(2+\frac{8 M^{2}}{W^{2}}-\frac{16 M^{4}}{W^{4}}\right) \ln \frac{W+\sqrt{W^{2}-4 M^{2}}}{2 M}-\sqrt{1-\frac{4 M^{2}}{W^{2}}}\left(1+\frac{4 M^{2}}{W^{2}}\right)\right] .
$$

This equivalent-photon approach is simpler than a full QED calculation in that it neglects the virtuality of the intermediate photon lines in Fig. 1. For this reason, we do not consider $e^{+} e^{-}$pairs, where this intermediate state affects the pair $p_{T}$ distribution [5, 10]. 


\section{TWO-PHOTON REACTIONS ACCOMPANIED BY NUCLEAR BREAKUP}

Because $Z \alpha$ is large, two-photon reactions may be accompanied by additional photonuclear reactions, as in Fig. (1b). As long as the two meson-producing photons do not excite the nucleus that emitted them, this is the dominant diagram for producing a meson while exciting both nuclei. As with photoproduction of vector mesons accompanied by MCD [7, 9], the kinematics of photon emission does not favor nuclear excitation. Then, the individual photon reactions are independent [20] and the processes factorize [21]. Photonuclear breakup can be incorporated in Eq. ([6) by including the photonuclear excitation probability, $P(b)$ :

$$
\frac{\mathcal{L}_{\gamma \gamma}}{d W d y}=\mathcal{L}_{A A} \frac{W}{2} \int_{b_{1}>R_{A}} d^{2} b_{1} \int_{b_{2}>R_{A}} d^{2} b_{2} n\left(k_{1}, b_{1}\right) n\left(k_{2}, b_{2}\right) P(b)\left[1-P_{H}(b)\right]
$$

where $b=\left|\vec{b}_{1}-\vec{b}_{2}\right|$. Here, $P(b)$ is the total breakup condition of interest, whether it is one nucleus or two. The two-photon luminosity accompanied by single nuclear excitation was calculated in this approach in Ref. [22]. We separately consider two cases. The first, labelled $X n$ covers all nuclear excitation, including high-energy excitations which may emit pions in addition to dissociating the nucleus. The second, single neutron emission, $1 n$, is a subset of $X n$, in which a single neutron is emitted.

The lowest order probability for Coulomb breakup of a specific nucleus is

$$
P_{X n}^{1}(b)=\int_{E_{\min }}^{E_{\max }} d k \frac{d^{3} n_{\gamma}}{d^{2} b d k} \sigma_{\gamma A \rightarrow A^{*}}(k) .
$$

where $k$ is the photon energy and $d^{3} n_{\gamma} / d^{2} b d k$ the photon density from Eq. (3). $\sigma_{\gamma A \rightarrow A^{*}}(k)$, the excitation cross section, is determined by data collected over a wide range of energies [23]; $E_{\min }$ is the minimum energy for this excitation, while $E_{\max }=\gamma \hbar c / b$ is the maximum photon energy for which there is significant flux. The superscript 1 shows that this is the lowest order probability. A similar calculation was performed in Ref. [24].

More precisely, $P_{X n}^{1}(b)$ is the mean number of excitations; the probability of having exactly $\mathrm{N}$ excitations follows a Poisson distribution, so the probability for at least one Coulomb excitation is

$$
P_{X n}(b)=1-\exp \left(-P_{X n}^{1}(b)\right)
$$

Single neutron emission usually occurs when the nucleus is excited into a giant dipole resonance (GDR), where the protons and neutrons oscillate against each other [25]. Photons 
with energies from 8-24 MeV can excite a GDR resonance in heavy nuclei. To observe the $1 n$ breakup, the GDR excitation must not be accompanied by any higher-energy reactions:

$$
P_{1 n}(b)=P_{1 n}^{1}(b) \exp \left(-P_{X n}^{1}(b)\right)
$$

The lowest order probability $P_{1 n}^{1}(b)$ is determined as in Eq. (12), except that the photon energy integration is truncated at the maximum GDR energy, $24 \mathrm{MeV}$ for both nuclei. Data on photoemission of single neutrons is used, avoiding uncertainties in the GDR branching ratios [26].

In MCD, the two nuclear breakups occur independently [4, 21, 23], so the probability for MCD is

$$
P_{X n X n}(b)=\left(P_{X n}(b)\right)^{2}
$$

and

$$
P_{1 n 1 n}(b)=\left(P_{1 n}(b)\right)^{2} .
$$

This independence has been verified by comparing the cross-sections for single nuclear breakup, both $1 \mathrm{n}$ and Xn, and MCD [27]. It also appears to hold for $\rho^{0}$ photoproduction with accompanying MCD [7].

\section{CROSS SECTIONS, RAPIDITY AND MASS DISTRIBUTIONS}

Table I shows the two-photon widths and cross sections for the different tags at RHIC and the LHC, for several meson resonances and for $\mu^{+} \mu^{-}$and $\tau^{+} \tau^{-}$, from Eq. (11). For a 'standard' RHIC year, the $550 \mu \mathrm{b}$ cross-section for the $f_{2}(1270)$ leads to 1,100,000 events/year. At the LHC, a $1 \mu \mathrm{b}$ cross section corresponds to 1,000 events/year.

Untagged meson production rates have been calculated by many authors [1, 15, 29, 30, 31]. Unfortunately, some authors used different ion species and Lorentz boosts. Several earlier papers considered uranium beams at RHIC with $\gamma=100$. These papers found $\eta^{\prime}$ production cross-sections of $2.9 \mathrm{mb}$ [35] to $3.6 \mathrm{mb}$ [30]. For lead at the LHC, with $\gamma=4000$ (rather than the planned $\gamma=2940$ which is used here), they find $\eta^{\prime}$ cross-sections of 30 and $32 \mathrm{mb}$ respectively, compared to the $22 \mathrm{mb}$ found here. Roldao and Natale [31] consider uranium beams at RHIC, and lead beams with $\gamma=3360$ at the LHC, using the Cahn-Jackson

approach [18]; our RHIC results are about 40\% lower than theirs; the LHC results are quite comparable, except that our $\eta_{c}$ cross-section is 6 times larger than theirs. It is difficult 
TABLE I: Spin/parity, two-photon widths $\Gamma_{\gamma \gamma}$ [28], and cross sections for two-photon production of various final states with and without nuclear breakup.

\begin{tabular}{|c|c|c|c|c|c|}
\hline Meson & $J^{P C}$ & $\begin{array}{r}\Gamma_{\gamma \gamma} \\
(\mathrm{keV})\end{array}$ & $\begin{array}{l}\text { overall } \\
\sigma[\mathrm{mb}]\end{array}$ & $\begin{array}{l}\mathrm{XnXn} \\
\sigma[\mu \mathrm{b}] \\
\end{array}$ & $\begin{array}{r}\ln 1 \mathrm{n} \\
\sigma[\mu \mathrm{b}]\end{array}$ \\
\hline \multicolumn{6}{|c|}{ Gold beams at RHIC } \\
\hline$\eta$ & $0^{-+}$ & $0.510 \pm 0.026$ & 1.05 & 51.7 & 4.9 \\
\hline$\eta^{\prime}$ & $0^{-+}$ & $4.30 \pm 0.15$ & 0.72 & 49.7 & 4.6 \\
\hline$f_{2}(1270)$ & $2^{++}$ & $2.60 \pm 0.24$ & 0.55 & 44.9 & 4.1 \\
\hline$f_{2}^{\prime}(1525)$ & $2^{++}$ & $0.081 \pm 0.009$ & 0.0069 & 0.63 & 0.057 \\
\hline$\eta_{c}$ & $0^{-+}$ & $7.2 \pm 0.7 \pm 2.0$ & 0.0029 & 0.40 & 0.034 \\
\hline$\mu^{+} \mu^{-}$ & & & 142 & 5,628 & 537 \\
\hline$\tau^{+} \tau^{-}$ & & & 0.00079 & 0.14 & 0.011 \\
\hline \multicolumn{6}{|c|}{ Lead beams at LHC } \\
\hline$\eta$ & $0^{-+}$ & $0.510 \pm 0.026$ & 18.8 & 337 & 21.6 \\
\hline$\eta^{\prime}$ & $0^{-+}$ & $4.30 \pm 0.15$ & 21.9 & 469 & 30.3 \\
\hline$f_{2}(1270)$ & $2^{++}$ & $2.60 \pm 0.24$ & 23.4 & 562 & 35.7 \\
\hline$f_{2}^{\prime}(1525)$ & $2^{++}$ & $0.081 \pm 0.009$ & 0.38 & 9.7 & 0.62 \\
\hline$\eta_{c}$ & $0^{-+}$ & $7.2 \pm 0.7 \pm 2.0$ & 0.57 & 19.3 & 1.2 \\
\hline$\mu^{+} \mu^{-}$ & & & 2,017 & 33,084 & 2,128 \\
\hline$\tau^{+} \tau^{-}$ & & & 0.55 & 22.8 & 1.4 \\
\hline
\end{tabular}

to understand the large difference for the $\eta_{c}$. The agreement between these calculations is generally good, but detailed comparisons are not possible.

Newer calculations have used the same species and boosts. Our cross-sections are 0 to $20 \%$ higher than those in Ref. [36]; this work seems to use $\gamma=100$ for RHIC. The exception is $\eta_{c}$ production at RHIC, where the current cross-section is 6 times higher. The difference may lie in how the papers handle the non-collision requirement; Bertulani and Navarra apply a form factor to the nuclei instead of applying an explicit requirement that $b>2 R$; this can lead to substantially different results near the kinematic limit.

These rates are also very similar to the $\eta_{c}$ results reported in Ref. [29]. Our rates are 
10-25\% higher than those in a very similar calculation [15]; the difference stems from slightly different $R_{A}$ (Ref. [15] used an A-dependent parameterization of the nuclear radius, rather than electron scattering data [12]) and older values for the two-photon widths, $\Gamma_{\gamma \gamma}$.

The RHIC $\mu^{+} \mu^{-}$and $\tau^{+} \tau^{-}$rates are about $20 \%$ and $33 \%$ respectively lower than in Ref. [29]. The $\tau$ pairs are close to threshold, so are very sensitive to the detailed of the nuclear surface calculation. For the $\mu$ pairs, the difference may be because Ref. [29] integrates over rapidity rather than the impact parameter for the second photons; the effect on the nuclear and final-state overlap requirements are not transparent. The RHIC and $\mathrm{LHC}^{+} \mu^{-}$ rates are also about $37 \%$ and $22 \%$ respectively lower than approximate Born calculations of Hencken, Kuraev and Serbo [37] at roughly the same energies ( $\gamma$ of 108 and 3000). One of us has also performed Born calculations by numerical integration [38], and found results consistent with Hencken, Kuraev and Serbo when adjusted for the slightly lower energies of the numerical calculations ( $\gamma$ of 100 and 2760). These Born calculations have no cutoff in integration over $b_{1}$ and $b_{2}$; point charges are assumed for the ions and a correction is made by applying a form factor for the virtual photons emanating from each ion.

Notable differences between the various calculations are in the treatment of the possibility of accompanying hadronic interactions and in how the virtual photons are cut off, either by integration over $b_{1}$ and $b_{2}$ or by a form factor. Here, the ultimate accuracy depends on a knowledge of the nuclear matter distribution; although there are accurate measurements of the proton distributions in heavy nuclei. Data on neutron radii is sparse, but it appears that neutrons are less confined than protons in heavy nuclei. For lead the neutron radius has been estimated to be $0.17 \mathrm{fm}$ larger [32]. Figure 3 shows how $\mathcal{L}_{\gamma \gamma}$ varies with $W$ for a $+/-10 \%$ change in nuclear radius, for gold beams at RHIC. A $\pm 10 \%$ change in radius leads to a $\pm 10-30 \%$ change in luminosity. Uncertainty in the nuclear radius leads to an irreducible systematic uncertainty in $\gamma \gamma$ luminosity.

A similar limitation holds for $\gamma \gamma$ interactions with protons, where the choice of proton form factor or radius can substantially affect the photon flux [39]. Efforts to use lepton pair production to measure the luminosity at the LHC [40] have to take into consideration these radius and form factor uncertainties as well as the possibility of higher order Coulomb corrections arising from the large $\mathrm{Z}$ heavy ions [2].

The $X n X n$ and $1 n 1 n$ cross-sections in Table I are new. They are about 1/10 and 1/100 of the untagged cross sections, respectively, at RHIC; at the LHC the reduction is a bit 


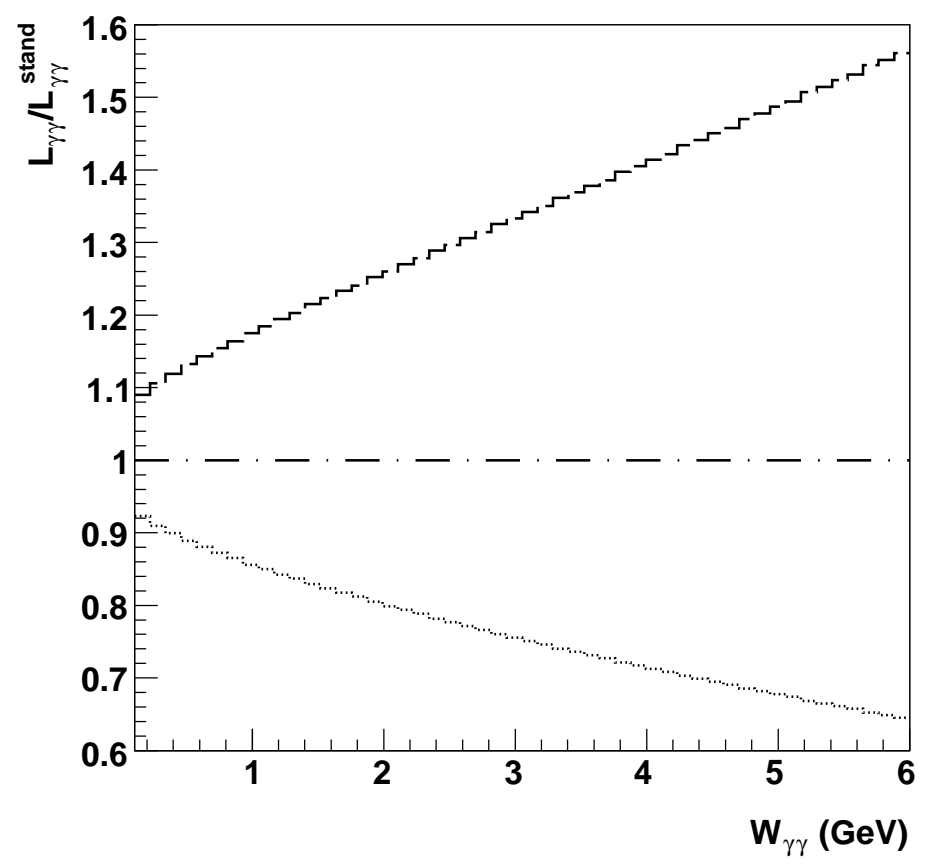

FIG. 3: Two-photon luminosity $\mathcal{L}_{\gamma \gamma}$ as a function of $W$ for two different nuclear radii $(+/-10 \%$ change) for gold beams at RHIC.

larger. These are small fractions, but $X n X n$ reactions may still be of value because of the triggering advantages that they provide.

The cross sections and corresponding production rates for single mesons are high. For example, one can expect to produce $\approx 10^{6}$ and $\approx 23 \cdot 10^{6} f_{2}(1270)$ mesons in a standard RHIC and LHC year, respectively. If one requires mutual Coulomb breakup (XnXn) the rates are reduced to $\approx 90,000$ and $\approx 560,000$. The $\eta_{c}$ might be hard to detect at RHIC, but the rates should be sufficient at the LHC, where around 600,000 $\eta_{c}$ mesons should be produced in one year. Of these, $\approx 20,000$ should remain if one requires XnXn breakup. Although these rates are high, it should be noted that the cross section for coherent vector meson production with similar event topology are about two order of magnitudes larger for mesons of similar mass [9, 12]. A way to separate the two reactions may be through the different $p_{T}$-spectra, as will be discussed in the next section.

Figure 4 shows the rapidity distribution $d \sigma / d y$ for $f_{2}(1270)$ and $\eta_{c}$ production at RHIC and the LHC. Spectra for $X n X n$ and $1 n 1 n$ breakup are shown, along with the untagged $d \sigma / d y$. The distributions with breakup are slightly narrower than those without. This is 

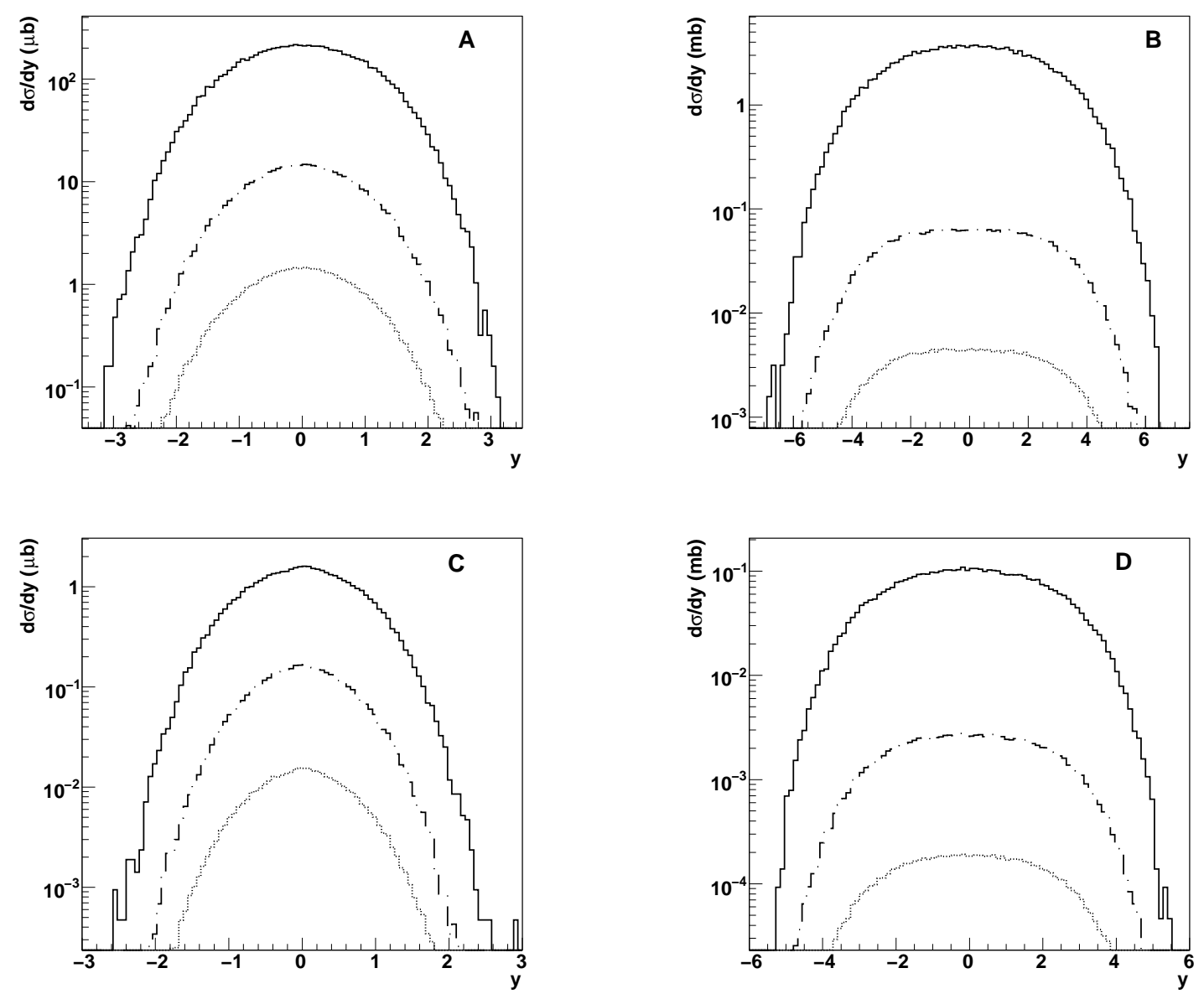

FIG. 4: The rapidity spectrum $d \sigma / d y$ for $f_{2}(1270)$ with (a) gold beams at RHIC and (b) lead beams at the LHC, and for the $\eta_{c}$ with (c) gold at RHIC and (d) lead at the LHC. Three curves are shown in each panel: the total two-photon cross section (solid curve), and with $X n X n$ (dashed curve) and $1 n 1 n$ (dotted curve) excitation.

because MCD selects events with smaller impact parameters. This increases the fraction of events with hard photons, eliminating interactions at large impact parameter, where a low energy photon far from it's source nucleus interacts with a higher energy photon near it's source; the large energy disparity corresponds to a large rapidity.

Figure 5 compares the $\mu^{+} \mu^{-}$and $\tau^{+} \tau^{-}$pair mass distributions at RHIC and the LHC. Subject to the aforementioned caveats about beam species and energies, the untagged spectra are similar to those found by other authors [1, 29, 41]. The solid line shows the untagged spectra, while the dashed and dotted lines are for $X n X n$ and $1 n 1 n$, respectively. The mass spectra with breakup are harder than those without. 

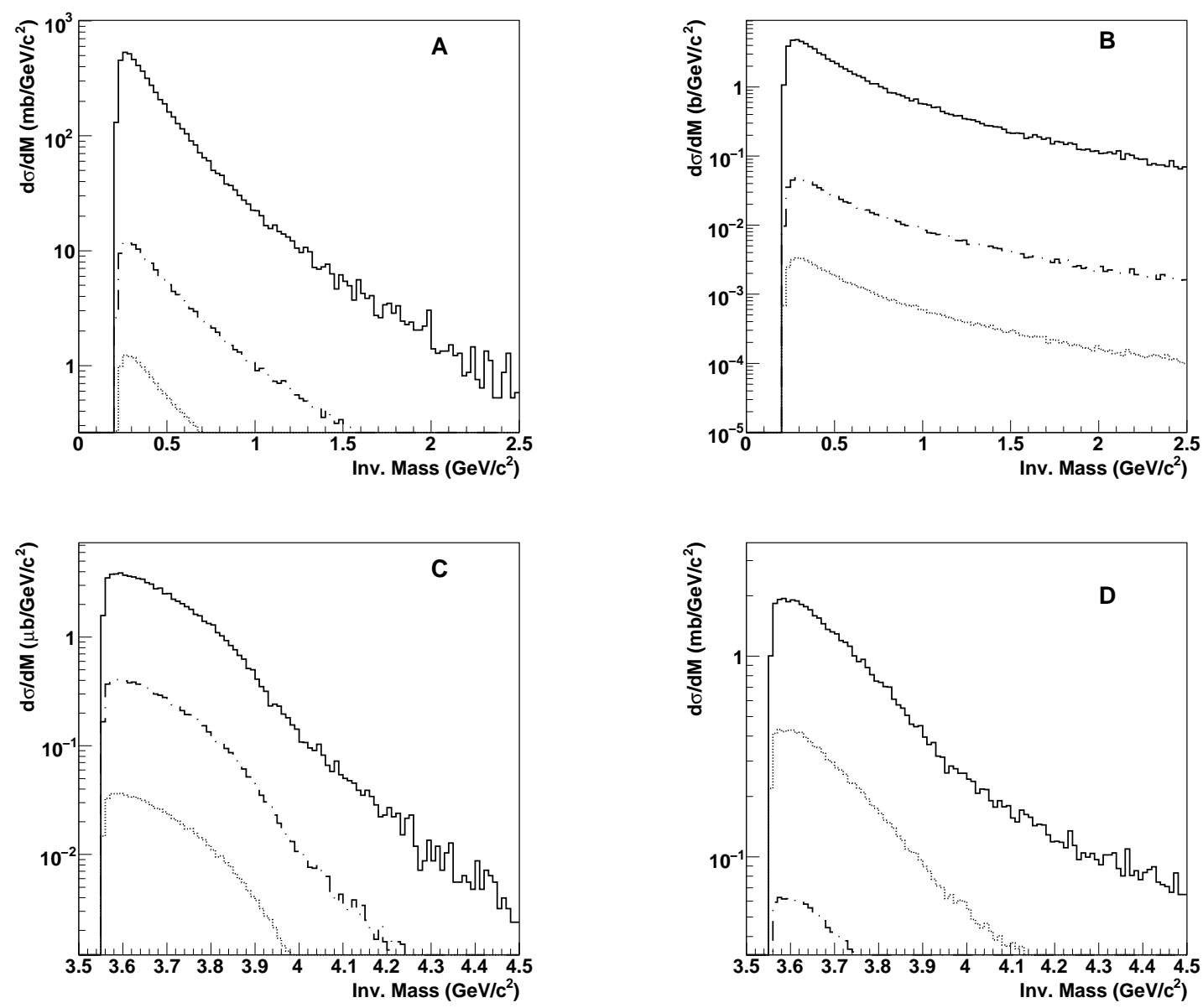

FIG. 5: The invariant mass spectrum $d \sigma / d M$ for $\mu^{+} \mu^{-}$pairs with (a) gold beams at RHIC and (b) lead beams at the LHC, and for the $\tau^{+} \tau^{-}$with (c) gold at RHIC and (d) lead at the LHC. Three curves are shown in each panel: the total two-photon cross section (solid curve), and with $X n X n$ (dashed curve) and $1 n 1 n$ (dotted curve) excitation.

\section{TRANSVERSE MOMENTUM SPECTRA}

Ultra-peripheral interactions (UPCs) are fully coherent with both nuclei, and so have a very small final state $p_{T}$; this characteristic is important for separating UPCs from background events. Here we consider a more complicated problem, that of separating fully reconstructed $\gamma \gamma$ interactions from coherent photonuclear interactions. The final state meson $p_{T}$ is the vector sum of the two photon perpendicular momenta, $k_{\perp}$. We assume that, integrated over all of transverse space this angular distribution is flat, and add the two-photon $k_{\perp}$ in quadrature. 
At a distance $b$ from the center of the emitting nucleus the photon flux is [19]

$$
\frac{d^{3} n(b, k)}{d k d^{2} b}=\frac{Z^{2} \alpha}{\pi^{2} k}\left|\int_{0}^{\infty} d k_{\perp} \frac{F\left(k_{\perp}^{2}+k^{2} / \gamma^{2}\right) k_{\perp}^{2}}{k_{\perp}^{2}+k^{2} / \gamma^{2}} J_{1}\left(b k_{\perp}\right)\right|^{2}
$$

where $J_{1}$ is a Bessel function.

For heavy nuclei, the nuclear charge form factor $F$ can be analytically modelled by the

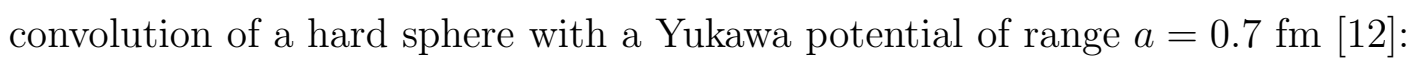

$$
F(q)=\frac{4 \pi \rho_{0}}{A q^{3}}\left[\sin \left(q R_{W S}\right)-q R_{W S} \cos \left(q R_{W S}\right)\right]\left(\frac{1}{1+a^{2} q^{2}}\right)
$$

Here, $\rho^{0}$ is the density and $R_{W S}$ is the Woods-Saxon radius of the nucleus. This gives an excellent approximation to the Woods-Saxon distribution. The radii of the heavy ions are well measured however recently published results indicate that the neutron and proton distributions differ in the nuclei which may lead to a larger radii of the matter distribution and essentially limit the accuracy of the photon flux determination [32].

The $k_{\perp}$ spectra of the virtual photon fields are complicated because $k_{\perp}$ and transverse position are conjugate variables, and cannot both be defined at the same time. This complicates any determination of the $k_{\perp}$ spectrum with constraints on transverse position, such as those imposed by photoexcitation reactions. We avoid this problem by selecting the photon energies, and then determining the transverse momenta solely by using the energies, using Eq. (17). The photon $k_{\perp}$ spectrum for fixed $k$ is given by [33],

$$
\frac{d N}{d k_{\perp}}=\frac{2 Z^{2} \alpha F^{2}\left(k_{\perp}^{2}+k^{2} / \gamma^{2}\right) k_{\perp}^{3}}{\pi\left[k_{\perp}^{2}+k^{2} / \gamma^{2}\right]^{2}}
$$

This is the same approach used to calculate $p_{T}$ spectra for vector meson photoproduction [33]. Hencken and collaborators used Eq. (19), with a point-particle form factor and a cutoff $p_{T}<1 / R_{A}$ [22]. Other groups (including some of the present authorship) used this equation with a Gaussian form factor [34].

The photon $k_{\perp}$ distribution is asymmetric, with a maximum at $k_{\perp}=\sqrt{3} k / \gamma$ and large tail at high $k_{\perp}$. The distribution has two different momentum scales, $\hbar / R_{A}$ and $k / \gamma$. For $\hbar / a>k_{\perp} \gg \hbar / R_{A}$ and $k_{\perp} \gg k / \gamma$, the tail falls as $1 / k_{\perp}^{5}$, regulated by the form factor.

The final state $p_{T}$ is the vector sum of the two photon $k_{\perp}$. We assume that the angle between the two photons is completely random, neglecting the possibly different cross sections for photons with parallel and perpendicular spin. 


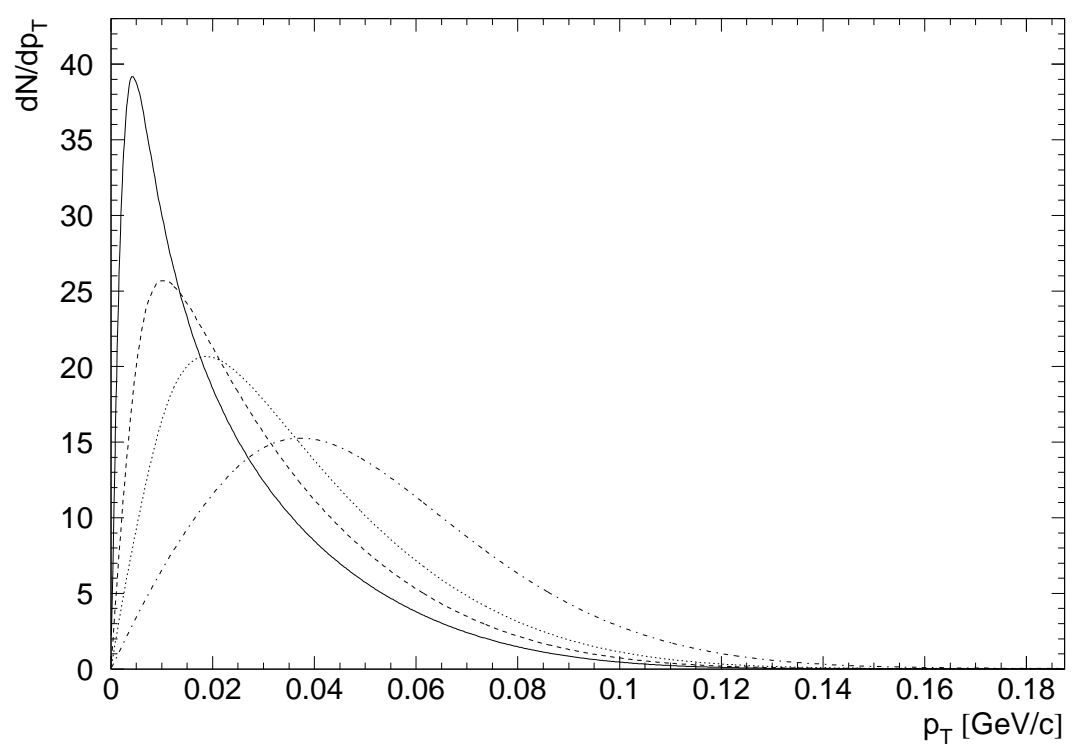

FIG. 6: The transverse momentum spectrum $d N / d p_{T}$ at mid-rapidity, $y=0$, for two-photon production of states with masses of 0.2 (solid), 0.5 (dashed), 1.0 (dotted) and 3.0 (dash-dotted) $\mathrm{GeV}$ in $\mathrm{Au}+\mathrm{Au}$ interactions at RHIC. The curves are normalized to have equal areas [42].

Figure 6] shows the $p_{T}$ spectra at mid-rapidity, $y=0$, for two-photon production of states with masses of $0.2,0.5,1.0$ and $3.0 \mathrm{GeV}$ in $\mathrm{AuAu}$ collisions at RHIC. The spectra peak around $\sqrt{1.5} \mathrm{M} / \gamma$. The average $p_{T}$ is around $75 \%$ larger, because of the long high- $p_{T}$ tail.

In photonuclear interactions, most of the $p_{T}$ comes from the nucleus; the typical $p_{T}$ is is a few $\hbar / R_{A}$, or $50-100 \mathrm{MeV} / \mathrm{c}$ [7, 9]. This is several times higher than is typical for two-photon interactions. Particularly at the LHC, where $M / \gamma$ is very small, $p_{T}$ should be an effective tool for separating the two event classes.

\section{CONCLUSIONS}

We have calculated the total cross sections and rapidity and transverse momentum distributions for two-photon production of various final states with and without nuclear breakup. The cross-sections for $\gamma \gamma$ interactions accompanied by $X n X n$ and $1 n 1 n$ dissociation are about $1 / 10$ and $1 / 100$ of that for the unaccompanied $\gamma \gamma$ interactions. The nuclear breakup tagged events have different rapidity and $p_{T}$ distributions from the un-tagged events, and 
can be used to explore the effects of different photon spectra and $b$ distributions. The typical $p_{T}$ of $\gamma \gamma$ final states is several times smaller than for comparable coherent photonuclear interactions, so $p_{T}$ cuts may be an effective tool for separating the two interactions.

This work was supported by the U.S. Department of Energy under Contracts No. DEAC-03076SF00098 and DE-AC02-98CH10886.

[1] C. A. Bertulani, S. R. Klein and J. Nystrand, Ann. Rev. Nucl. Part. Sci. 55, 271 (2005). G. Baur, K. Hencken, D. Trautmann, S. Sadovsky and Y. Kharlov, Phys. Rep 364, 359 (2002); G. Baur, K. Hencken and D. Trautmann, J. Phys. G 24, 1657 (1998); F. Krauss, M. Greiner and G. Soff, Prog. Nucl. Part. Phys. 39, 503 (1997).

[2] A. J. Baltz et al., Phys. Rept. 458, 1 (2008).

[3] K. Hencken, D. Trautmann and G. Baur, Phys. Rev. C53, 2532 (1996).

[4] A.J. Baltz, C. Chasman and S. N. White, Nucl. Instrum. \& Meth. A417, 1 (1998).

[5] J. Adams et al., Phys. Rev. C70, 031902 (2004).

[6] V. Morozov, PhD Dissertation, UC Berekeley, 2003, preprint nucl-ex/0403002.

[7] C. Adler et al., Phys. Rev. Lett. 89, 272303 (2002); B. I. Abelev et al., Phys. Rev. C77, 034910 (2008); B. I. Abelev et al., preprint arXiv:0812.1063.

[8] S. Afanasiev et al., preprint arXiv:0903.2041.

[9] A. Baltz, S. R. Klein and J. Nystrand, Phys. Rev. Lett. 89, 012301 (2002).

[10] K. Hencken, G. Baur and D. Trautmann, Phys. Rev. C69, 054902 (2004).

[11] A. J. Baltz, Phys. Rev. Lett. 100, 062302 (2008).

[12] S. R. Klein and J. Nystrand, Phys. Rev. C60, 014903 (1999).

[13] S. J. Brodsky, T. Kinoshita and H.Terazawa, Phys. Rev. D4, 1532 (1971).

[14] V. M. Budnev, I. F. Ginzburg, G. V. Meledin and V. G. Serbo, Phys. Rep. 15, 181 (1974).

[15] J. Nystrand and S. Klein, nucl-ex/9811007.

[16] C. G. Roldao and A. A. Natale, Phys. Lett. B581, 161 (2004).

[17] G. Baur and L.G. Ferreira Filho, Nucl. Phys. A518, 786 (1990).

[18] R. N. Cahn and J. D. Jackson, Phys. Rev. D42, 3690 (1990).

[19] M. Vidovic, M. Greiner, C. Best and G. Soff, Phys. Rev. C47, 2308 (1993); M. Vidovic, M. Greiner and G. Soff, Phys. Rev. C48, 2011 (1993). 
[20] S. N. Gupta, Phys. Rev. 99, 1015 (1955).

[21] G. Baur, et al., Nucl. Phys. A729, 787 (2003).

[22] K. Hencken, D. Trautmann and G. Baur, Z. Phys. C C68, 473 (1995).

[23] A. J. Baltz, M. J. Rhoades-Brown and J. Weneser, Phys. Rev. E54, 4233 (1996).

[24] I. A. Pshenichnov et al., Phys. Rev. C64, 024903 (2001).

[25] M. Goldhaber and E. Teller, Phys. Rev. 74, 1046 (1948).

[26] K. Alder et al., Rev. Mod. Phys. 28, 432 (1956).

[27] M. Chiu et al., Phys. Rev. Lett. 89, 012302 (2002).

[28] C. Amsler et al., Phys. Lett. B667, 1 (2008).

[29] M. Vidovic, M. Greiner and G. Soff, J. Phys. G. 21, 545 (1995).

[30] K. A. Chikin et al., Eur. Phys. J A8, 537 (2000).

[31] C. G. Roldao and A. A. Natale, Phys. Rev. C61, 064907 (2000).

[32] A. Trzcinska et al., Phys. Rev. Lett. 87, 082501 (2001).

[33] S. R. Klein and J. Nystrand, Phys. Rev. Lett. 84, 2330 (2000).

[34] S. Klein and E. Scannapieco, STAR Note 243, 1995 (unpublished); available at http://drupal.star.bnl.gov/STAR/starnotes/public/sn0243

[35] G. Baur and L.G. Ferreira Filho, Nucl. Phys. A518, 786 (1990).

[36] C. Bertulani and F. Navarra, Nucl. Phys. A703, 861 (2002).

[37] K. Hencken, E. A. Kuraev and V. G. Serbo, Phys. Rev. C 75, 034903 (2007).

[38] A. J. Baltz, arXiv:0901.0891 [nucl-th].

[39] S. Klein and J. Nystrand, Phys. Rev. Lett. 92, 142003 (2004).

[40] A. Abulencia et al., Phys. Rev. Lett. 98, 112001 (2007).

[41] G. Baur, Z. Phys. C 54, 419 (1992).

[42] J. Nystrand, preprint nucl-th/0112055. 